\title{
Highly Nonlinear Fiber as Pre-amplifier in Distributed Fiber Raman Amplifier Bus Networks for Sensors Multiplexing
}

\author{
Silvia Diaz ${ }^{\mathrm{a}}$, Manuel Lopez-Amo ${ }^{\mathrm{a}}$, Paul Urquhart ${ }^{\mathrm{a}}$, César Jáuregui ${ }^{\mathrm{b} *}$ and José Miguel López-Higuera ${ }^{\mathrm{b}}$ \\ ${ }^{a}$ Dep. of IEE, Public University of Navarra, Campus de Arrosadía s/n, 31006 Pamplona, Spain \\ ${ }^{b}$ Dep.TEISA, University of Cantabria, Avda. los Castros s/n, 39005 Santander, Spain \\ * now at ORC, $U K$ \\ silvia.diaz@unavarra.es
}

\begin{abstract}
We experimentally compare two distributed fiber Raman amplified bus networks for the wavelength-division multiplexing of optical sensors. The main difference between them is the type of fiber used in the pre-amplifier in each case.

(C)2006 Optical Society of America

OCIS codes: (060.4230) Multiplexing; (050.2770) Gratings; (060.2370) Fiber optics sensors; (140.4480) Optical amplifiers; (190.5650) Raman effect.
\end{abstract}

\section{INTRODUCTION}

Bus architectures are some of the most widely used multiplexing topologies for sensors, mainly due to their simple cabling requirements [1]. For example, a wavelength-division-multiplexed (WDM) optical fiber-based network configured as a linear bus has been applied as a means of addressing and subsequently gathering data from optical sensors [2]. It consists of a spine section that connects a series array of directional couplers leading to the sensing elements, followed by fiber Bragg gratings (FBGs).

Nevertheless, fiber bus networks suffer from the disadvantage that the optical power is reduced along the bus and it limits the number of sensors that can be addressed at acceptable signal-to-noise ratios (SNRs). One approach to overcome this restriction is to locate optical amplifiers (such as erbium doped fiber amplifiers, EDFAs) within the bus, but they are costly devices that require electrical power supplies. Alternatively, one can fabricate the bus entirely from erbium-doped fiber with a low doping density to provide distributed gain, but it requires special (and potentially expensive) fiber [2]-[3] and the working bandwidth is limited.

The use of Raman amplification in the spine section of the bus to maintain the received powers from the sensors within acceptable bounds has recently been reported [4]. Moreover, dual-wavelength pumped Raman amplification, together with an EDFA, has been applied in a long-distance sensing system using a fiber Bragg grating [5]-[6].

In our work we compare two topologies consisting of a Raman bus structure, but introducing two different types of fiber: dispersion compensating fiber (DCF) and a highly nonlinear fiber (HNLF). We show how both Raman gain and signal-to-noise ratios can be increased by using the second configuration.

\section{NETWORK CONFIGURATION}

Both configurations consist of a bus topology, as shown in Fig. 1, with an active bus built from standard single-mode fiber (ITU-G.652 compliant). They use wavelength-division multiplexing (WDM) for the identification of four sensors. Each sensor incorporates a narrow-bandwidth FBG at a unique wavelength. The launched signals are ultimately incident on all of the sensors but the gratings ensure that each sensor returns only its characteristic channel towards the launching point (the head end) after passing through the sensor a second time. The peak wavelengths and reflectivities of the gratings are marked on Fig. 1 . They had a wavelength variation with temperature of $0.01 \mathrm{~nm} /{ }^{\circ} \mathrm{C}$.

We used $90 \%$ directional couplers to perform power distribution among the sensors. Their insertion loss varied from $10.4 \mathrm{~dB}$ to $10.8 \mathrm{~dB}$ in the $10 \%$ branch for the pump and the signal wavelengths and from $0.5 \mathrm{~dB}$ to $0.7 \mathrm{~dB}$ in the $90 \%$ branch for the pump and the signal wavelengths.

In all of our demonstrations, the sensors were removed in order to make the power measurements independent of the particular measurands and so ensure greater generality of the results. Therefore, although the gratings themselves may be used as sensors, the network is not designed to be specific to any particular type of sensor. The Raman pump, signal(s) and receivers are co-located in one head end. Therefore, the Raman pump propagated co-directionally with the launched signal but contra-directionally with the retuned signals from the gratings. All of the free terminations on the bus were 


\section{ThE52.pdf}

refractive-index-matched to frustrate unwanted reflections. This is very important to minimize noise due to multi-path interference [7].

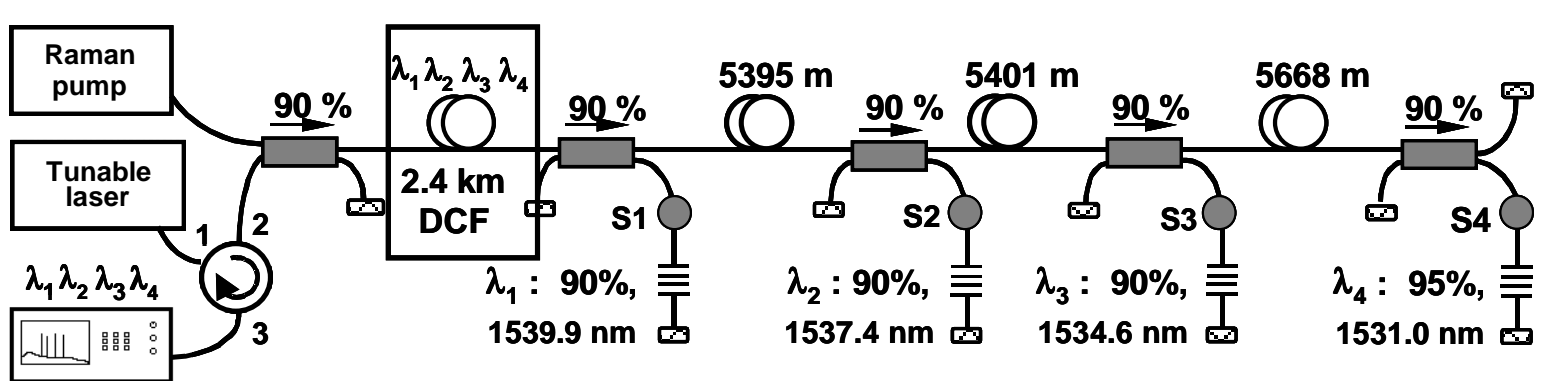

(a)

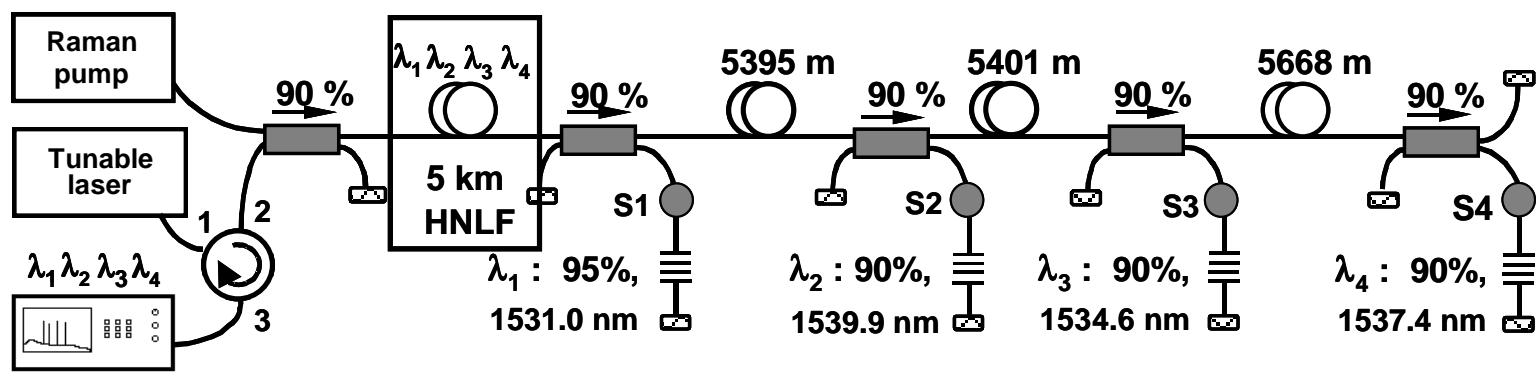

(b)

Fig. 1. (a) Wavelength-division-multiplexed distributed fiber Raman amplifier bus network adding $2.4 \mathrm{~km}$ of dispersion compensating fiber (DCF) at the head end of the bus. S1-S4: location of sensors. (b) Improved distributed fiber Raman amplifier bus network, adding $5 \mathrm{~km}$ of highly nonlinear fiber (HNLF) at the head end of the bus. S1-S4: location of sensors. The fiber lengths and the grating peak wavelengths and reflectivities are indicated. Taking into account the circulator loss, the launched signal power at port 2 is $-2.6 \mathrm{dBm}$ in both cases.

The pump laser that we used was a multi-order fiber Raman laser and it radiated in three lines, $1428 \mathrm{~nm}, 1445 \mathrm{~nm}$ and $1466 \mathrm{~nm}$. The signal was provided by a tunable laser $(1460-1580 \mathrm{~nm})$ and after passing through the launch circulator, it had a power of $-2.6 \mathrm{dBm}$ and a spectral linewidth of $5 \mathrm{MHz}$. The launched pump was polarization scrambled but there was a small residual elliptical polarization of the signal laser. By taking multiple measurements, +/$0.5 \mathrm{~dB}$ errors are estimated in the gain values.

The original experimental configuration of Fig. 1(a) [8] has been modified by replacing the initial span of DCF (of length $2.4 \mathrm{~km}$ ) with $5 \mathrm{~km}$ of highly nonlinear fiber (HNLF), as shown in Fig. 1(b). Dispersion compensating fiber shows relatively high Raman amplification, however, the low power conversion efficiency is considered to be a major difficulty that must be overcome [9]. In order to enhance the gain of the Raman amplifier, we have proposed highly nonlinear fiber (HNLF) with a Raman gain coefficient of $2.2 \mathrm{~W}^{-1} \mathrm{~km}^{-1}$ and attenuation of lower than $0.4 \mathrm{~dB} / \mathrm{km}$ around $1550 \mathrm{~nm}$. Moreover, DCF has a raised germania content and therefore a somewhat higher loss coefficient than SMF. We have measured a fiber loss of $0.55 \mathrm{~dB} / \mathrm{km}$ for the DCF. The availability of new high-power optical sources in the $1550-\mathrm{nm}$ wavelength range as well as the development of highly nonlinear fibers (HNLF) offer increased possibilities to design devices relying on nonlinear effects in optical fibers [10].

From the theory of Raman amplification it is known that fibers with a relatively high effective area, such as SMF, have a low pump power conversion efficiency and therefore as distributed amplifiers they are oriented to long span networks. However, with the judicious use of HNLF it is possible to achieve greater flexibility in bus lengths without having to launch excessive pump powers. HNLF is a good Raman gain medium, due to its small effective core area $(9$ $\mu \mathrm{m}^{2}$ compared with $16 \mu \mathrm{m}^{2}$ for the DCF). As shown in Section III, we obtained the best results with this fiber. 


\section{ThE52.pdf}

\section{RESULTS}

In order to evaluate an amplifying network, we define a bus spine transparency power. This is the value of the Raman pump power that is sufficient to overcome the signal attenuation due to (a) the fiber and (b) the discrete losses experienced at the couplers in passing from one amplifying span to the next. This power level does not include compensation for the $2 \times 10 \mathrm{~dB}$ losses in passing from the spine of the bus to the sensors and back again or for the losses of the launch coupler and circulator. Fig. 2 was obtained using the configuration of Fig. 1(a) with the Raman pump switched on.

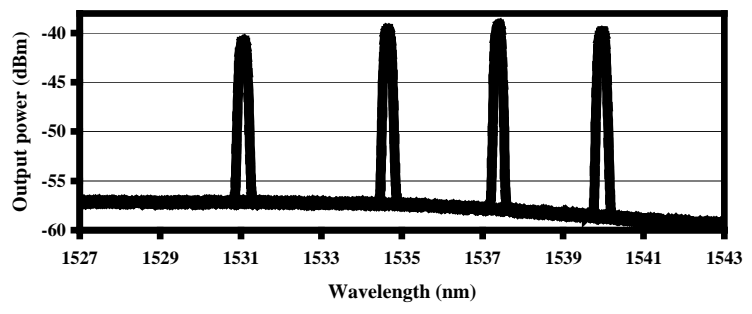

Fig. 2. Amplified output power obtained with an applied pump power of $270 \mathrm{~mW}$. The signal wavelengths are those of Fig. 1(a).

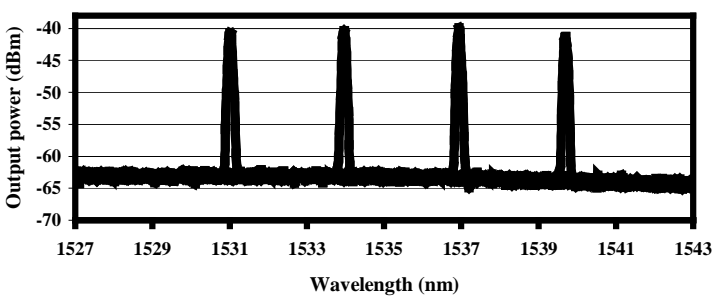

Fig. 3. Amplified output power obtained with an applied pump power of $250 \mathrm{~mW}$. The signal wavelengths are those of Fig. 1(b).

The power used in Fig. 2 was $270 \mathrm{~mW}$ because this is the bus spine transparency power for the return path to S4. The original experimental setup has been modified in order to obtain higher Raman gain. The improved fiber-bus is that of Fig. 1(b). S1... S4 show the positions the sensors ought to take in the new network, although these sensors have been removed in the interest of generalization, as already mentioned. In the improved bus structure, a pump power of $250 \mathrm{~mW}$ is sufficient to obtain the transparency condition due to the greater Raman gain efficiency when highly nonlinear fiber is used.

Fig. 3 is a plot of the amplified output power obtained with an applied pump power of $250 \mathrm{~mW}$. Clearly, there is a power increase in all channels and a marked improvement in channel power equalization. It is important to notice that with the new configuration the Raman gain profile has changed, as well as the gain level with respect to the original setup with the $2.4 \mathrm{~km}$ dispersion compensating fiber span.

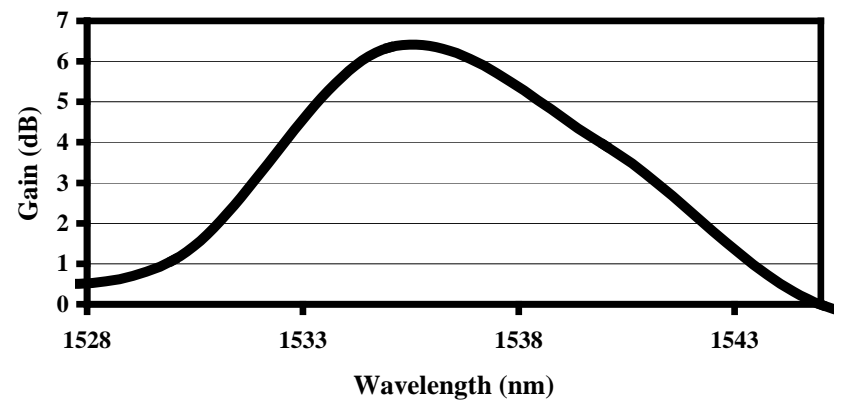

Fig. 4. New Raman gain profile for the HNLF fiber at $250 \mathrm{~mW}$.

Fig. 4 is a representation of the new Raman gain profile for a pump power of $250 \mathrm{~mW}$. In this way, by adding the 5 $\mathrm{km}$ of HNLF there is enough distance to obtain Raman gain for every grating, even for the one closest to the pump. We used this property to obtain a degree of equalization of the received powers from the four gratings. In both cases of Fig. 1 , the strategy we used was to place the gratings corresponding to low Raman gains closest to the pump because that is where the signals experience enough interaction lengths. Conversely, gratings with relatively high Raman gain wavelengths were located further from the head end. 


\section{ThE52.pdf}

TABLE I

MEASUREd VALUES OF POWER AND OpticAl Signal To NOISE RATIO OBTAINED FROM FIG. 2

\begin{tabular}{ccccc}
\hline \hline & \multicolumn{4}{c}{ FBG wavelengths } \\
\cline { 2 - 5 } & $\mathrm{S} 1$ & $\mathrm{~S} 2$ & $\mathrm{~S} 3$ & $\mathrm{~S} 4$ \\
\hline$\lambda, \mathrm{nm}$ & 1539.9 & 1537.4 & 1534.6 & 1531.0 \\
\hline $\begin{array}{c}\text { Pr (pumped), } \\
\text { dBm }\end{array}$ & -39.96 & -39.1 & -39.67 & -40.97 \\
\hline $\begin{array}{c}\text { OSNR } \\
\text { (pumped), } \\
\mathrm{dB}\end{array}$ & 18.8 & 18.6 & 17.7 & 16.1 \\
\hline \hline
\end{tabular}

TABLE II

MeAsured VALUES OF POWER AND OPTICAL Signal To NOISE RATIO OBTAINED FROM FIG. 3

\begin{tabular}{ccccc}
\hline \hline & \multicolumn{4}{c}{ FBG wavelengths } \\
\cline { 2 - 5 } & $\mathrm{S} 1$ & $\mathrm{~S} 2$ & $\mathrm{~S} 3$ & $\mathrm{~S} 4$ \\
\hline$\lambda, \mathrm{nm}$ & 1531.0 & 1539.9 & 1534.6 & 1537.4 \\
\hline $\begin{array}{c}\mathrm{Pr}(\text { pumped), } \\
\mathrm{dBm}\end{array}$ & -40.7 & -41.3 & -40.3 & -39.9 \\
\hline $\begin{array}{c}\text { OSNR } \\
\text { (pumped), } \\
\mathrm{dB}\end{array}$ & 22 & 22.1 & 22.1 & 22.9 \\
\hline \hline
\end{tabular}

Table I and Table II summarize the received optical powers, Pr, obtained from each of the four sensors, and their corresponding optical signal to noise ratios (OSNR) considering the original and the improved setups of Fig. 1(a) and 1(b), respectively. We had a minimum improvement of $3.3 \mathrm{~dB}$ for the sensor at $1539.9 \mathrm{~nm}$ and a maximum of $5.9 \mathrm{~dB}$ for the sensor at $1531 \mathrm{~nm}$. Better equalization is obtained when using the highly nonlinear fiber and also the optical signal to noise ratios are better when HNLF is used, as shown in Table II.

\section{CONCLUSIONS}

We have demonstrated an improved distributed Raman amplifying bus network for wavelength division multiplexing of sensors, which uses a highly nonlinear fiber. This structure yields greater absolute received signal power and better SNR values. The improvement is achieved by using only $270 \mathrm{~mW}$ Raman pump power. Our results obtained with high power pumping indicate that there is potential to extend the bus network to serve greater numbers of sensors.

\section{ACKNOWLEDGEMENTS}

Financial support from the Spanish Comisión Interministerial de Ciencia y Tecnología within project TEC200405936-C02 and FEDER is acknowledged.

\section{REFERENCES}

[1] A. Dandridge, C. Kirkendell, "Passive Fiber Optic Sensor Networks" in Handbook of Optical Fiber Sensing Technology, J.M. López-Higuera, ed. (John Wiley, 2002), Chap. 21.

[2] M. López-Amo, L. T. Blair and P. Urquhart, "Wavelength-Division-Multiplexed Distributed Optical Fiber Amplifier Bus Network for Data and Sensors," Opt. Let. 18, 1159-1161 (1993).

[3] R. Hernández-Lorenzo, M. López-Amo and P. Urquhart, "Single and Double Distributed Optical Amplifier Fiber Bus Networks with Wavelength Division Multiplexing for Photonic Sensors," J. Lightwave Technol. 16, 485-489 (1998).

[4] S. Diaz, G. Lasheras, M. López-Amo, P. Urquhart, C. Jáuregui and J. M. López-Higuera, "Wavelength-Division-Multiplexed Distributed Fiber Raman Amplifier Bus Network for Sensors", in Proc. 17th Int. Conf. Optical Fiber Sensor (OFS-17), Proc. SPIE, vol. 5855, (Bruges, 2005), pp. $242-244$

[5] J. H. Lee, Y. M. Chang, Y. G. Han et al., "Raman Amplifier-Based Long-Distance Remote, Strain and Temperature Sensing System Using an Erbium-Doped Fiber and a Fiber Bragg Grating," Opt. Express 12, 3515-3520 (2004).

[6] Y. Nakajima, Y. Sindo and T. Yoshikawa, "Novel concept as long-distance transmission FBG sensor system using distributed Raman amplifier," in Proc. 16th Int. Conf. Optical Fiber Sensor (OFS-16), (Japan, 2003), pp. 530-533.

[7] J. Bromage, P. J. Winzer, R. J. Essiambre, "Multiple Path Interference and its Impact on System Design," in Raman Amplifiers for Telecommunications 2, M. N. Islam, ed. (Springer, 2004), Chap. 15.

[8] S. Diaz and M. Lopez-Amo, "Comparison of wavelength-division-multiplexed distributed fiber Raman amplifier networks for sensors," Opt. Express 4, 1401-1407.

[9] T. Amano, K. Okamoto, T. Tsuzaki, M. Kabui and M. Shigematsu, "Hybrid dispersion compensating Raman amplifier module employing highly nonlinear fiber," in Proc. Int. Optical Fiber Communications Conference (OFC 2003), (Atlanta, 2003), pp. 306-307.

[10] J. Hansryd, F. Dross, M. Westlund, P. A. Andrekson and S. N. Knudsen, "Increase of the SBS threshold in a short highly nonlinear fiber by applying a temperature distribution," J. Lightwave Technol. 19, 1691-1697 (2001). 